\title{
Julia Eckert*
}

\section{Tugend, Recht und Moral: Tendenzen der Verantwortungszuschreibung}

DOI 10.1515/dzph-2016-0018

\begin{abstract}
The attribution of responsibility in world society is increasingly a field of contestation. On the one hand, the perceptions of far-reaching causal and moral links between spatially and temporally distant events are ever more explicitly pronounced; on the other hand, the very complexity of these links often engenders a fragmentation of responsibility in law as well as in moral commitment. Identifying three competing conceptualisations of responsibility, namely a turn to virtue ethics, processes of juridification, and processes of moralisation, this article explores their different temporal and social dimensions, and the effects they each have on the relation between those held responsible and those affected by the situation that is to be accounted for.
\end{abstract}

Keywords: virtue, law, charity, responsibility, social anthropology

Die Zuschreibung von Verantwortung in der Weltgesellschaft ist zunehmend ein Feld sozialer Kämpfe. Auf der einen Seite werden globale Vernetzungen, kausale Zusammenhänge und moralisch relevante Interdependenzen immer deutlicher wahrgenommen; auf der anderen Seite führt die Komplexität dieser Verbindungen vielfach zu einer Fragmentierung von Verantwortung. Dies gilt für rechtliche Verantwortung; ${ }^{1}$ aber auch die den globalen Verflechtungen angemessenen moralischen Normen von Verantwortung sind alles andere als eindeutig. Die Frage, ob und welche „Verantwortungsbegriffe“ gegenwärtig Schlüsselbegriffe unserer Gesellschaft sind, möchte ich aus sozialanthropologischer Perspektive deswegen dahingehend spezifizieren, ob und welche Veränderungen in der Zuschreibung von Verantwortung in unterschiedlichen Zusammenhängen zu beobachten sind und welche Muster sich in solchen Veränderungen erkennen lassen. Ich werde die These verfolgen, dass gegenwärtig drei Vorstellungen von Verantwortung Überzeugungskraft haben, die ich als Hinwendung zu tugendethi-

1 Vgl. Veitch (2007).

*Kontakt: Julia Eckert, Universität Bern, Institut für Sozialanthropologie, Lerchenweg 36, 3000 Bern 9, Schweiz; julia.eckert@anthro.unibe.ch 
scher Selbstverantwortung, als Juridifizierung und als Moralisierung von Verantwortung beschreibe. Diese drei Verantwortungskonzeptionen unterscheiden sich in Reichweite und Qualität der sozialen Beziehung, die sie stiften, nämlich in ihrem Begriff von „Verursachung“, d. h., wie umfassend die zeitlichen und räumlichen Dimensionen der einen Sachverhalt hervorbringenden Handlungszusammenhänge in ihnen abgebildet sind, sowie dem Verhältnis von Freiwilligkeit und Pflicht.

Um nachzeichnen zu können, welche Vorstellungen von Verantwortung sich heute in unterschiedlichen Kontexten durchsetzen, verwende ich einen Begriff von Verantwortung, der es erlaubt, in einer vergleichenden Perspektive unterschiedliche Bewegungen oder auch normative Orientierungen als Ausdruck verschiedener Verantwortungskonzeptionen zu beschreiben. ${ }^{2}$ Ein solch breiter Verantwortungsbegriff liegt aus sozialanthropologischer Sicht nahe. Verantwortung als Zurechnung einer spezifischen Verpflichtung einer Person gegenüber anderen hinsichtlich eines Sachverhalts auf Grund kausaler, ethischer oder rechtlicher Annahmen ist aus sozialanthropologischer Perspektive kein zeithistorisches Phänomen, sondern eine Schlüsseloperation jeder Gesellschaft und für jede Gesellschaftsordnung grundlegend. Wie aber solche Zurechnung sozial organisiert ist, variiert: Unterschiedliche Verantwortungskonzeptionen unterscheiden sich in den Wissenssystemen von Kausalität, der Verknüpfung von Verursachung und Haftung, den Rechten und Pflichten, die sie auferlegen, den Gründen, die zu Schuldfähigkeit wie auch zu Schuldunfähigkeit oder eingeschränkter Haftung führen, und den moralischen Grundannahmen, die soziale Verantwortungsbeziehungen begründen. In Verantwortungskonzeptionen drücken sich also verschiedene Personenbegriffe aus. Sie enthalten Annahmen über die Handlungsmächtigkeit und Schuldfähigkeit von Individuen und Kollektiven. Sie unterscheiden sich dahingehend, wem (oder was) „moral agency“ zugeschrieben wird, welche Rolle Intentionalität spielt, ob oder in welchem Maße ein Mensch in seinem

2 Der Streit darüber, ob es zulässig ist, unterschiedliche Formen der sozialen Organisation der Zurechnung von Verpflichtungen einer Person oder einer Gruppe gegenüber anderen auf Grund kausaler, ethischer oder rechtlicher Annahmen, die jeweils ihre eigene terminologische Bezeichnung haben, unter dem Begriff der Verantwortung zu fassen, bzw. ob sie damit korrekt übersetzt sind, wird letztlich seit der Debatte zwischen Paul Bohannan und Max Gluckman (vgl. ihre Beiträge in Nader 1969) geführt und ist bis heute offen. Allerdings ist eine vergleichende Perspektive ohne eine solche Übersetzung nicht möglich; nur sie erlaubt, Unterschiede und Gemeinsamkeiten und deren Bedingungen und Effekte zu thematisieren. Jedes Postulat terminologischer Authentizität verpflichtete uns letztlich auf eine deskriptive Bestandsaufnahme und verschlösse so auch die Möglichkeit, Veränderungsprozesse von Institutionen in Hinblick auf ihre Angleichung oder Differenzierung zu untersuchen. 
Handeln durch freie Entscheidung oder aber durch andere Kräfte - seien dies Hormone, Geister, Karma oder aber die sozialen Bedingungen - geleitet wird und welche Rolle dies für Haftung und Verpflichtung spielt. Verantwortungskonzeptionen machen also explizit, welche Vorstellung von Individualität diesen Ordnungen zugrunde liegt und wie Aspekte wie Alter, Lebensphasen, Geschlecht, Status, Annahmen über Intentionen, Emotionen oder klinische Befunde zu neurologischen Zuständen Grade der Zurechnungsfähigkeit bestimmen. Oft ist der heute dominante Personenbegriff vom autonomen Individuum für seine eurozentrische Perspektive kritisiert worden. Doch ist die Frage weniger, ob es einen freien Willen gibt, als welche Rolle die Annahme desselben in unterschiedlichen Verantwortungskonzeptionen einnimmt. Das Prinzip der verschuldensunabhängigen Haftung z. B. ist überall zu finden. ${ }^{3}$ Die Zuschreibung von Verantwortung ist also in gewissem Sinne unabhängig von den Befunden zur Existenz oder NichtExistenz des freien Willens bzw. des autonomen Individuums; sie ist vielmehr eine soziale Beziehungen konstituierende und definierende Operation.

Für die gegenwärtige Diskussion von der Globalisierung angemessenen Verantwortungsnormen erscheint die Bewertung der Kapazität, für sein eigenes Handeln Rechenschaft abzulegen, allerdings weit weniger zentral als die Unterschiede verschiedener Verantwortungskonzeptionen hinsichtlich der zeit-räumlichen Dimensionen, die sie umfassen, und die die rechtsrelevante Reichweite von Handlungsketten in Vergangenheit und Zukunft sowie sozial bestimmen. Die Verantwortungsbeziehungen, die in die Vergangenheit oder in die Zukunft entworfen werden, sind in vielen traditionellen Ordnungen weitreichender als z. B. im modernen Recht. Interessanterweise erscheinen auch die Konzepte der Verursachung vielfach als sozial umfassender als moderne Konzepte der Verursachung. So hat Stuart Kirsch die Vorstellungen von Haftung unterschiedlicher melanesischer Gruppen mit denen verglichen, die von transnationalen Unternehmen auf Grundlage wissenschaftlich erfasster Kausalzusammenhänge sowie darauf aufruhender rechtlicher Haftpflichtvorschriften vorgebracht werden. ${ }^{4}$ Während die von ihm diskutierten melanesischen Haftungsnormen den zur Verantwortung ziehen, der den Kontext für eine bestimmte soziale Interaktion, die zu Schäden geführt hat, hergestellt hat, begrenzen wissenschaftliche Gutachten zu Verursachung Haftbarkeit auf unmittelbare Verursacher. Moderne Institutionen der Verantwortung, so könnte man diese Beobachtung auf den Punkt bringen, erfassen nicht die Ermöglichung von Handeln; ihre Begriffe der Beihilfe oder Unterstützung z. B. sind meist eng, und daher sind die institutio-

3 Vgl. Falk-Moore (1972).

4 Vgl. Kirsch (2001). 
nellen „Schnitte“" , die sie in den Handlungsketten vornehmen, inkongruent mit alternativen Wahrnehmungen kausaler Zusammenhänge und den daraus resultierenden Zuschreibungen von Verantwortung. ${ }^{6}$

Wie weit in Raum und Zeit diese Verknüpfungen reichen, ist nicht nur in verschiedenen Normordnungen unterschiedlich, sondern verändert sich innerhalb ihrer: Verantwortungsbeziehungen können schrumpfen oder expandieren. Verantwortungsgemeinschaften, wie ich es gerne nennen würde, sind insofern auch immer Felder der Inklusion und Exklusion, und ihre Ausdehnung ist oft umkämpft: Genau dies scheint gegenwärtig Kern vieler Auseinandersetzungen um Verantwortungszuschreibung, wie sie in Kämpfen um Klimagerechtigkeit, globale Produktions- und Konsumptionsketten oder auch um Verantwortung in Gewaltkonflikten zum Ausdruck gebracht werden. ${ }^{7}$

\section{Tugendethische Verantwortung}

Als ich 1997 begann, in Indien die Beziehungen zwischen Hindus und Muslimen in jenen Gebieten zu erforschen, die von Gewaltausschreitungen betroffen gewesen waren, traf ich unter den Muslimen viele, die sich der Tabligh-Bewegung zugehörig fühlten, einer inzwischen weltweit unter sunnitischen Muslimen verbreiteten, puritanischen Bewegung. Viele waren der Bewegung nach den Pogromen gegen Muslime in Bombay im Jahr 1993 beigetreten. Sie wurde als der richtige Weg wahrgenommen, weil sie sich als apolitisch darstellte und somit einen nach den Gewalterfahrungen für viele Muslime beängstigenden konfrontativen Kurs sowie

5 Strathern (1996).

6 Bruno Latour hat die Verlängerung von Netzwerken bzw. Handlungsketten in der Moderne aufgezeigt und auf die auf diese Netzwerke verteilte Agency hingewiesen (vgl. Latour 1995). Marilyn Strathern (1996) hat seinerzeit auf Latours These geantwortet, dass zwar eventuell moderne Netzwerke bzw. Handlungsverkettungen weiterreichend geworden seien, dass aber moderne Institutionen wie z. B. das moderne Eigentumsrecht diese Netzwerke und die darin konstituierten sozialen Beziehungen „abschnitten“, und zwar in weit kürzere Abschnitte als viele traditionelle Institutionen. Während nicht-moderne Eigentumskonzepte die Sozialität von Eigentum berücksichtigten, verkürzten moderne Eigentumskonzepte diese. Sie verweist auf das Beispiel des intellektuellen Eigentumsrechts und seine Privilegierung der „Erfindung“ bzw. Innovation, die gerade von den durch Latour betonten Netzwerken, die zu technischen Innovationen führen, abstrahierten und ein Patent nur dem „Erfinder“ zuschreibt, nicht aber denen, die am Gesamtprozess der Entwicklung beteiligt waren. Diese Spezifik moderner institutioneller Schnitte kann man, folgt man z. B. Kirsch (2001), auch auf Institutionen der Verantwortung übertragen.

7 Vgl. Sundar (2004) und Clarke (2010). 
jedwede Forderung an die Mehrheitsgesellschaft vermied. Diese apolitische Positionierung begründeten die Tablighis in Bombay damit, dass die Muslime eigentlich selbst für die enorme Gewalt verantwortlich seien, der sie ausgesetzt gewesen waren. Nicht, dass sie den Kreislauf der Gewalt losgetreten oder die Hindus provoziert hätten - nein, Gott hätten sie provoziert und die Gewalt sei als kollektive Strafe für ihre Verfehlungen gegenüber Gott über sie gekommen. Kausal ursächlich war also ihr eigenes Verhalten in der Vergangenheit gegenüber Gott, d. h. die Verletzung ihrer religiösen Pflichten. Verantwortlich für die Folgen waren somit sie selber. Die Verantwortungsbeziehung war die zwischen Gott und jedem einzelnen.

Ich interpretierte damals diese Zuschreibung von Verantwortung als eine Form, mit der eigenen Ohnmacht umzugehen, um angesichts der politischen Unmöglichkeit, von den Tätern Gerechtigkeit zu erlangen, die Eigenverantwortung für das Leiden ins Zentrum zu stellen und so auch wieder Handlungsmacht zu erhalten - denn wo man selber verantwortlich war, konnte man auch selber Abhilfe leisten. Dementsprechend schlugen die Anhänger der Tabligh-Bewegung auch ganz spezifische Strategien ein: Es ging um Selbstverbesserung, individuelle Frömmigkeit und um die Erfüllung der Verpflichtungen gegenüber Gott. Verantwortung zu übernehmen hieß hier, die Pflege des Selbst nach spezifischen Regeln zu unternehmen. Eine tugendethische Orientierung wurde zur Grundlage einer Selbstermächtigung, die der Person Handlungsmacht in Hinblick auf die Kontrolle ihres Geschickes zusprach.

Eine solche Hinwendung zur Tugendethik und der daraus folgenden Selbstverantwortlichung begegnet einem nicht nur unter Gläubigen der abrahamitischen Religionen und hängt somit wohl nicht nur mit deren spezifischen Theodizee-Vorstellungen zusammen. So beobachtete David Engel in den späten 1990er-Jahren einen drastischen Rückgang der Versicherungsklagen bei Autounfällen in Thailand. ${ }^{8}$ Er suchte die Opfer der Unfälle auf und fand, dass sie die Ereignisse nicht mehr im Sinne der Schuld der Fahrer interpretierten, sondern als Ausdruck der Kräfte von lokalen Geistern und als ihr eigenes Verschulden, diese Geister nicht in Betracht gezogen zu haben. Sie machten sich bzw. ihr Karma dafür verantwortlich, an Orte und in Situationen geraten zu sein, in denen sie Verletzungen erfuhren. Auch hier war also eine Transformation sowohl im Kausalitätsnarrativ als auch in der daraus folgenden Verantwortungszuschreibung zu beobachten; auch hier folgte der Transzendentalisierung des Kausalitätsnarrativs die Individualisierung der Verantwortung.

8 Vgl. Engel (2010). 
Joel Robbins ${ }^{9}$ beobachtet allgemein die Zunahme solcher Verantwortungsmodelle in neuen religiösen Bewegungen von puritanischer, millenaristischer oder fundamentalistischer Orientierung und weist auf die ihnen allen gemeinsame Fokussierung auf das eigene Verschulden, die Versündigung durch Regelverletzung hin, die zum zentralen ethischen Maßstab wird. Was von Robbins auf Grund der Bedeutung der Schuld durch religiöse Pflichtverletzung als deontologische Ethik beschrieben wird, ist genauer vielleicht als Tugendethik zu verstehen, denn neben der Vermeidung von Pflichtverletzungen oder allgemein von ,Sünde“ liegt die Aufmerksamkeit in all diesen Bewegungen ebenso zentral in der Entwicklung eines tugendhaften Selbst. Astrid Bochow und Rijk van Dijk verwenden den Begriff ,responsabilization' und weisen auf die kirchlichen Institutionen hin, die über neue Pädagogiken und Diskurse unter den Anhängern der Pfingstkirchen spezifische Normen persönlicher und sozialer Verantwortung zu verankern versuchen und sie in spezifischen Auffassungen der moralischen Person ausbilden. ${ }^{10}$ Diese neuen Vorstellungen einer moralisch guten Person sind eng verknüpft mit (religiösen) Praktiken der „Arbeit am Selbst“, insbesondere Abstinenz und Fleiß.

Die konkreten Umstrukturierungen sozialer Beziehungen, die durch diese neuen Normen von Verantwortung erreicht werden, sind weitreichend: Sowohl unter den Muslimen Bombays als auch unter den Pfingstkirchenanhängern in Malawi, Ghana oder Botswana bedeutet die Hinwendung zur Tugendethik die Herauslösung aus bestehenden sozialen Zusammenhängen, die Verschiebung von Fürsorgepflichten von Verwandtschaftsverbänden oder anders begründeten Solidargemeinschaften zu Kleinfamilien, ${ }^{11}$ von Ahnenkulten zur individuellen Heilserwartung, ${ }^{12}$ die Abkehr von synkretistischen und regionalen Festen $\mathrm{zu}$ bewegungsspezifischen Ritualen. ${ }^{13}$ Wir beobachten hier eine Veränderung sowohl der sozial-räumlichen als auch der zeitlichen Dimensionen von Verantwortung, die das Individuum und dessen Zukunft ins Zentrum der Produktion einer moralisch guten Person stellt. Auch wenn sich die Zukunftskonzeptionen im Einzelnen unterscheiden, so ist es doch die binäre Beziehung zwischen Gott und dem Frommen, die hier das Zukunftsgeschehen bestimmt und dieses in die Verantwortung, somit aber auch in die Kontrolle der Frommen, verlagert. ${ }^{14}$

9 Vgl. Robbins (2010).

10 Vgl. Bochow/van Dijk (2012).

11 Vgl. van Dijk (2002).

12 Vgl. ebd.

13 Vgl. Eckert (2012).

14 Vgl. Robbins (2010). 
Man könnte diese tugendethischen Orientierungen und die in ihnen vonstattengehende Individualisierung und Selbstermächtigung unter Umständen als spezifische Variationen der Selbstverantwortlichung betrachten, und diese somit als ein sehr viel breiteres Phänomen als von Gouvernementalitätsstudien ${ }^{15}$ angedacht - und ich denke, es würde sich lohnen, hier über Familienähnlichkeiten nachzudenken.

\section{Die Juridifizierung von Verantwortung}

Doch sehen wir gleichzeitig eine andere Tendenz der Zuschreibung von Verantwortung, die ich als „Juridifizierung von Protest“ beschrieben habe. ${ }^{16}$ Damit bezeichne ich Bewegungen, die über die Berufung auf Recht bzw. auf dem Rechtswege spezifische Haftungs- und Fürsorgepflichten einklagen oder moralische Verantwortungsnormen rechtlich bindend machen wollen. Während die Tablighi die eigenen Verfehlungen kausal ursächlich und die Gläubigen individuell selbstverantwortlich für die erlittene Gewalt machten, kämpften manche ihrer muslimischen Nachbarn dafür, die Täter vor Gericht zu bringen. ${ }^{17}$ Nach den Pogromen von 2002 ging es ihnen $u$. a. darum, den heutigen Premierminister Indiens, Narendra Modi, vor den Internationalen Strafgerichtshof zu bringen. Diese Versuche scheiterten, ${ }^{18}$ aber dies ist hier nicht Thema. In diesen Versuchen - Verantwortung über das Strafrecht festzuschreiben - war in Indien eine relativ deutliche Fokussierung auf die Befehlsverantwortlichen zu beobachten; ähnlich wie das internationale Strafrecht, aber aus anderen Gründen, forderten in Indien viele, nicht die „kleinen Fische“, sondern vor allem jene zur Verantwortung zu ziehen, die als Veranlasser und Profiteure gesehen wurden und in deren Macht es gestanden hätte, die Gewalt zu unterbinden.

Die Fokussierung des Internationalen Strafgerichtshofs auf Befehlsverantwortung ist vielfach als reduktionistisch kritisiert worden. ${ }^{19}$ Dieser Reduktionismus im Konfliktnarrativ erscheint aus einer Perspektive, die sich auf die zeit-räumlichen und sozialen Dimensionen der von unterschiedlichen Verantwortungskonzeptionen als relevant erachteten Handlungsketten konzentriert, als spezifische Selektion der potentiell rechtsrelevanten „Beteiligungsformen“ an einem Sachver-

15 Vgl. Rose (1999).

16 Eckert et al. (2012).

17 Eckert (2012).

18 Indien hat das Römische Statut nie unterzeichnet.

19 Vgl. Clarke (2010) u. Anders (2012). 
halt. Die Kämpfe, die in solchen Juridifizierungsprozessen ausgetragen werden, betreffen die Frage, welche Handlungszusammenhänge rechtsrelevant und welche Positionen darin wie gewichtet werden, welche spezifischen Entscheidungen oder Handlungen also die unterschiedlichen Positionen in welchem Umfang als ursächlich für den Umstand bewerten, der zu verantworten ist. Die Kämpfe gehen darum, wo genau wie viel Verantwortung anzusiedeln ist, ob bei den Entscheidungsträgern oder den Ausführenden, denen, die von den problematischen Zusammenhängen profitieren, oder all jenen, die irgendwie an ihnen teilhaben und sie als Zulieferer, Konsumenten oder gar nur als Dulder, wie Judith Shklar sie thematisiert hat, ${ }^{20}$ ermöglichen. Dies sind Fragen, die insbesondere in Hinblick auf die unendlich komplexen Handlungsketten in globalen Produktions- und Konsumptionsketten verhandelt werden: Welche internationalen oder globalen Verantwortungsstrukturen ergeben sich aus der Gemengelage der lokalen Produktion eines durch ein transnationales Unternehmen vertriebenen, global konsumierten Gutes? Welche Verantwortung trage ich für die Produktionsbedingungen in Bangladesch, wenn ich bei H\&M einkaufe? Letztlich aber sind diese Produktions- und Konsumptionsketten eben auch aufs Engste mit den Gewaltkonflikten verflochten, die über das internationale Strafrecht verhandelt werden. ${ }^{21}$

In Juridfizierungsprozessen geht es heute also darum, Verflechtungen, Interdependenzen und Beteiligungen genau zu benennen und jeder Person ihren Anteil an Verantwortung zuzuweisen, (fast) alle aber rechtlich relevant zu machen - wie dies in den oben erwähnten ,traditionellen“ Rechtsordnungen zum Teil gehandhabt wird. Juridifizierungsprozesse operieren mit der Annahme oder der Fiktion, dass fast jede Form der Beteiligung an einem Sachverhalt auch einen Aspekt der Verursachung enthält, und dass solche Verursachung zu gewissen Graden von

20 Vgl. Shklar (1990).

21 Interessant sind hier Konzepte wie die der „Unterstützung“ oder der „Beihilfe“. Der Versuch, über das Konzept der Beihilfe weiteren Handlungszusammenhängen gerecht zu werden und die ,Herstellung der Möglichkeit‘ als relevant für Verantwortungszuschreibung zu institutionalisieren, wird derzeit in verschiedenen Feldern noch äußerst unterschiedlich verwendet: In Fragen der Unterstützung terroristischer Netzwerke muss z. B. der Vorsatz zur Unterstützung einer terroristischen Tat nicht notwendig nachgewiesen werden. Jedwede, auch humanitär motivierte Sachleistung an Organisationen, deren Leistungen eventuell zur Legitimität als terroristisch klassifizierter Gruppen beitragen können, reicht auch ohne direkten Bezug zur Straftat aus, um von Unterstützung auszugehen (vgl. Schiffauer 2011, 180-192). Im Zusammenhang mit den wirtschaftlichen Aktivitäten von Rohstoffunternehmen in Konfliktgebieten werden Beihilfe und Unterstützung hingegen meist - zumindest erscheint es der Ethnologin so - eng ausgelegt. In diesen Fällen wird nur dann von Beihilfe gesprochen, wenn die der Straftat förderliche Handlung vorsätzlich deren Gelingen unterstützt. Wann ein solcher Vorsatz zur Beihilfe identifiziert oder als relevant angesehen wird, ist aber wiederum höchst unterschiedlich. 
Haftung führen muss. Sofern sie jedes Handeln als „,verursachend“ behandeln (wollen) und darüber Verantwortung zuschreiben, sind sie in der Begründung von Verantwortungsnormen konsequentialistisch.

Grundsätzlich kann man sagen, dass es in allen Juridifizierungsprozessen um vier Motive geht. Erstens gilt es, klare Zurechnung rechtlich bindend möglich zu machen. Zweitens soll sie gleichzeitig der Komplexität von Handlungszusammenhängen in einer Weltgesellschaft gerecht werden, um damit die Unzulänglichkeit einer nationalstaatlich verfassten Verantwortungsordnung zu überwinden. ${ }^{22}$ Drittens ist es auch das Ziel, das Spektrum von Verantwortlichkeit zu erweitern, indem Verantwortung für „Ermöglichung“, „Vorteil“ etc. über gegenwärtige Begriffe von Beihilfe und Unterstützung hinaus rechtlich bindend in Betracht gezogen werden kann. So wird viertens der emotionale oder affektive Gehalt von Verantwortung irrelevant: Entscheidend ist nie, ob einer sich verantwortlich fühlt oder ein schlechtes Gewissen hat. Der Juridifizierungsprozess ist „expansiv“; er kolonisiert gewissermaßen moralische und politische Verantwortung. Das heißt auch, dass Juridifizierung das Verhältnis von Freiwilligkeit und Pflicht im Begriff der Verantwortung ganz an den Pol der Pflicht verschiebt. Daraus entsteht eine spezifische Beziehung zwischen denen, die über solche Verantwortungszuschreibungen verknüpft werden: Der eine hat die Pflicht, der andere ein Recht, einen Anspruch.

\section{Die Moralisierung von Verantwortung}

Wir beobachten genau in diesem Zusammenhang einen dritten Typus von Verantwortungszuschreibung, der insofern vielleicht der interessanteste ist, als er zugleich Grundlage und Abwehr von Juridifizierungstendenzen sein kann. Es ist die Moralisierung von Verantwortung.

Die Moralisierung von Verantwortung ist sowohl eine Erweiterung als auch eine Verengung: Wie die Juridifizierung erweitert sie die Handlungszusammenhänge, für die sich ein Einzelner verantwortlich fühlen kann, beispielsweise Produktionsprozesse von H\&M-Socken. Zugleich entledigt die Moralisierung Verantwortung ihrer rechtlich bindenden Verpflichtungsaspekte und verschiebt Verantwortung in Richtung Barmherzigkeit. ${ }^{23}$ Die Moralisierung beginnt mit einem

22 Ich denke hier an Verantwortungsordnungen, wie sie z. B. Rawls (1999) entworfen hat, wie sie aber natürlich der Idee des Nationalstaats als Solidargemeinschaft inhärent sind (vgl. Beckert et al. 2004).

23 Vgl. Eckert (2013). 
konsequentialistischen Impetus und nimmt ähnliche Erweiterungen von Verantwortungsbeziehungen wie gegenwärtige Juridifizierungsprozesse vor. Doch solche Moralisierungsprozesse können sowohl in einer deontologischen Begründung von Verantwortung als auch in tugendethischen Orientierungen münden. Meine Vermutung wäre, dass deontologische Interpretationen ein Kontinuum zu Juridifizierungsprozessen herstellen, während tugendethische Orientierungen solchen eher entgegen stehen, denn es geht ihnen vornehmlich um die moralische Qualität der verantwortlichen Person („,der ethische Konsument“, „die faire Firma“), nicht um die Qualität der Beziehung zwischen Verantwortlichem und den vom Sachverhalt Betroffenen. Das (individuelle oder korporative) Gewissen wird zum zentralen Maßstab. Dies zeigt sich auch darin, dass die Übernahme solcher moralischer Verantwortung oft unspezifisch ist und Verantwortlichkeit von einem auf andere Sachverhalte verlagert: Man baut eine Schule, statt bessere Arbeitsbedingungen durchzusetzen etc.

Die Möglichkeit solcher Verlagerungen sowie die Freiwilligkeit der moralischen Verantwortung führen dazu, dass die Interdependenz derer, zwischen denen asymmetrische Verantwortungsbeziehungen bestehen, aus dem Blick verschwindet. Genau aus dieser Entkopplung entsteht wiederum eine spezifische Beziehung zwischen dem, der Verantwortung hat, und jenem, der vom Umstand betroffen ist, für den ersterer Verantwortung übernimmt: nämlich eine Beziehung, worin der eine freiwillig, aus moralischer Selbstverpflichtung „gibt“, der andere aber zum passiven Empfänger ohne Anrecht wird, der möglicherweise die Übernahme von Verantwortung durch den ersten durch Dankbarkeit oder eine spezifische Disposition entgelten muss. Handlungsmacht liegt allein bei dem, der Verantwortung trägt, der andere ist hilfsbedürftiger Empfänger.

Solche Moralisierung von Verantwortung zeigt sich als „Habitus“ weit über das Feld der Corporate Social Responsibility hinaus. Anthropologen wie Didier Fassin, Miriam Ticktin und andere sehen sie als Grundmerkmal unserer Zeit, als „humanitarian reason“24, als unsere gegenwärtige Disposition gegenüber Leiden, das wir lindern, statt seine Ursachen zu ändern. Sie weisen auf die Entpolitisierung hin, die sowohl in dem Verhältnis vom Geber zum Opfer enthalten ist, weil diese keinen Anspruch des Opfers enthält, als auch in den Nothilfemaßnahmen, die Symptome, nicht Ursachen bekämpfen. ${ }^{25}$ Freilich kann die Moralisierung auch Triebkraft der Politisierung sein. Doch geht sie - oft ungewollt - einher mit der Auflösung bindender (rechtlicher) Verantwortungsnormen, ersetzt sie, obwohl ohne eine solche Moralisierung auch die Erweiterung von Verantwor-

24 Fassin (2010).

25 Vgl. auch Rottenburg (2009). 
tungsbeziehungen durch Juridifizierungsprozesse wohl kaum möglich ist. In diesem Sinne muss die Moral dem Recht vorausgehen, das Recht aber folgt nicht notwendig der Moral.

\section{Schluss}

Allen drei Verantwortungskonzeptionen, der Hinwendung zur tugendethischen Selbstverantwortlichung, der Juridifizierung sowie der Moralisierung von Verantwortung, ist die Personalisierung, letztlich auch die Individualisierung von Verantwortung gemeinsam. Darin liegt das Spezifische all dieser Verantwortungsbegriffe im Vergleich zu anderen Konstitutionsprinzipien sozialer Beziehungen. Tugendethische Orientierung, Juridifizierung und Moralisierung unterscheiden sich also voneinander dahingehend, wie umfassend die Handlungszusammenhänge, die einen Sachverhalt hervorbringen, in ihnen abgebildet sind und welche Art der sozialen Beziehung sie stiften. Die unterschiedlichen Wirkungen dieser verschiedenen normativen Antworten auf die Transformation sozialer Beziehungen betreffen ihre soziale und temporale Ausdehnung sowie das Verhältnis zwischen Verantwortlichen und den vom zu verantwortenden Sachverhalt Betroffenen in Hinblick auf Rechte und Pflichten, Handlungsmacht und Souveränität. Gerade, weil diese verschiedenen normativen Entwürfe sich nebeneinander und teilweise als Alternativen zueinander entfalten, aber sehr unterschiedliche Auswirkungen auf soziale Beziehungen haben, gilt es zu verstehen, welche Chance sie in welchen Handlungsfeldern haben, verbindliche Institutionen auszubilden. Zu differenzieren wäre die These, derzufolge wir im Bereich bindender Verantwortungs- und Haftungsnormen immer noch eine Privilegierung physischer Gewalt beobachten, während im Bereich der Moral verhandelt wird, was man unter den Begriff struktureller Gewalt fassen könnte. Die etwa von Strathern thematisierten institutionellen Schnitte in Handlungszusammenhängen bzw. die engen oder weiten Begriffe von Verursachung (Unterstützung, Beihilfe) werden also abhängig von den spezifischen Zuordnungen oder Unterscheidungen zwischen physischer und struktureller Gewalt vollzogen. Diese Unterscheidung ist freilich selbst ein solcher institutioneller Schnitt durch die Handlungszusammenhänge, die Leid verursachen. 


\section{Literatur}

Anders, G. (2012), Juridification, Transitional Justice and Reaching Out to the Public in Sierra Leone, in: Eckert, J., Donahoe, B., Strümpell, C., u. Biner, Z. Ö. (Hg.), Law Against the State. Ethnographic Forays into Law's Transformation, Cambridge, 94-117.

Beckert, J., Eckert, J., Kohli, M., u. Streeck, W. (Hg.) (2004), Transnationale Solidarität. Chancen und Grenzen, Frankfurt am Main.

Bochow, A., u. van Dijk, R. (2012), Introduction: Christian Creations of New Spaces of Sexuality, Reproduction, and Relationships in Africa. Exploring Faith and Religious Heterotopia, in: Journal of Religion in Africa 42.4, 325-344.

Clarke, K. (2010), Rethinking Africa Through its Exclusions. The Politics of Naming Criminal Responsibility, in: Anthropological Quarterly 38.3, 625-651.

Eckert, J. (2012), Kultur und Schuld. Narrative der Verantwortung, in: Kaufmann, M., u. Renzikowsky, J. (Hg.), Zurechnung und Verantwortung, Baden-Baden, 155-167.

Eckert, J., Biner, Z. Ö., Donahoe, B., u. Strümpell, C. (Hg.) (2012), Introduction: Law's Travels and Transformations, in: dies. (Hg.), Law Against the State. Ethnographic Forays into Law's Transformations, Cambridge, 1-22.

Eckert, J. (2013), Ein Recht auf Fairness, in: Ich. Heute. 10 vor 8, URL: http://blogs.faz. net/10vor8/2013/11/18/ein-recht-auf-fairness-39/ (30.11.2015).

Engel, D. (2010), Tort, Custom, and Karma. Globalization and Legal Consciousness in Thailand, Stanford, Calif.

Falk-Moore, S. (1972), Legal Liability and Evolutionary Interpretation. Some Aspects of Strict Liability, Self-Help and Collective Responsibility, in: Gluckman, M. (Hg.), The Allocation of Responsibility, Manchester, 51-108.

Fassin, D. (2012), Humanitarian Reason. A Moral History of the Present, Berkeley, Calif.

Kirsch, S. (2001), Property Effects. Social Networks and Compensation Claims in Melanesia, in: Social Anthropology 9.2, 147-163.

Latour, B. (1995), Wir sind nie modern gewesen. Versuch einer symmetrischen Anthropologie, Frankfurt am Main.

Nader, L. (Hg.) (1969), Law in Culture and Society, Chicago.

Rawls, J. (1999), A Theory of Justice, Oxford.

Robbins, J. (2010), On the Pleasures and Dangers of Culpability, in: Critique of Anthropology 30, 122-128.

Rose, N. (1999), Powers of Freedom. Reframing Political Thought, Cambridge.

Rottenburg, R. (2009), Social and Public Experiments and New Figurations of Science and Politics in Postcolonial Africa, in: Postcolonial Studies 12.4, 423-440.

Schiffauer, W. (2014), Vor dem Gesetz. Der staatliche Umgang mit dem legalistischen Islamismus, in: Mecheril, P. (Hg.), Subjektbildung. Interdisziplinäre Analysen der Migrationsgesellschaft, Bielefeld, 165-183.

Shklar, J. (1990), Faces of Injustice, New Haven, Conn.

Strathern, M. (2001), Cutting the Network, in: Journal of the Royal Anthropological Institute 2, 517-535.

Sundar, N. (2004), Toward an Anthropology of Culpability, in: American Ethnologist 31.2, 145-163.

van Dijk, R. (2002), Religion, Pentecostalism and Restructuring Family Responsibility in the Ghanaian Pentecostal Diaspora, in: Bryceson, D., u. Vuorela, U. (Hg.), The Transnational Family, Oxford, 173-196.

Veitch, S. (2007), Law and Irresponsibility. On the Legitimation of Human Suffering, London. 